\title{
Conhecimento de mulheres da zona rural sobre o papilomavírus humano
}

\section{Knowledge of rural women about human papillomavirus}

\section{Luzia Soares Abreu' ${ }^{1}$ (i) Tamires Soares de Oliveira Andrade 2 (i) Zhilbelly Mota Nunes ${ }^{3}$ (i) Núbia Souza Rufino ${ }^{4}$ (1) Kaisy Pereira Martins ${ }^{5}$}

${ }^{1}$ Autora correspondência. Centro Universitário de João Pessoa (João Pessoa). Paraíba, Brasil. luziabreu195@gmail.com 2,3Universidade Federal da Paraíba (João Pessoa). Paraíba. Brasil. tami.andrade93@gmail.com, zhilbelly@hotmail.com ${ }^{4,5}$ Centro Universitário de João Pessoa (João Pessoa). Paraíba, Brasil. nubia_rufino@hotmail.com, kaisyjp@hotmail.com

RESUMO | OBJETIVO: Avaliar o conhecimento das mulheres de uma zona rural sobre o papilomavírus humano e formas de prevenção. MÉTODO: Estudo descritivo exploratório de abordagem qualitativa. Realizaram-se entrevistas semiestruturadas em outubro de 2017 com 43 mulheres cadastradas em uma Unidade de Saúde da Família localizada na zona rural do município de São João do Rio do Peixe. Utilizou-se a técnica de análise de conteúdo de Bardin. RESULTADOS: A partir dos dados obtidos na pesquisa emergiram três categorias: Conhecimento das mulheres sobre o papilomavírus humano (HPV); orientações recebidas na perspectiva das mulheres; e formas de prevenção utilizadas pelas mulheres contra o HPV. A compreensão sobre o HPV é permeada pela ausência de instrução acerca do vírus, embora a maioria das entrevistadas reconheça o preservativo como uma importante maneira de prevenção, mesmo relatando não terem recebido orientações adequadas sobre o HPV pela equipe da Unidade de Saúde da Família que atende na comunidade. CONCLUSÃO: Identificou-se déficit de conhecimento das mulheres da referida zona rural sobre o papilomavírus humano, principalmente quanto à forma de transmissão, suas causas e cuidados para a prevenção. Cabe à equipe de saúde desenvolver novas estratégias de educação em saúde sobre o HPV para que a comunidade tenha melhores informações relacionadas à prevenção da doença.

DESCRITORES: Papilomavírus humano. Prevenção. População Rural. Mulheres. Enfermagem.
ABSTRACT | OBJECTIVE: To evaluate women's knowledge in a rural area about human papillomavirus and ways of prevention. METHOD: A descriptive exploratory study with a qualitative approach. Semi-structured interviews were conducted in October 2017 with 43 women registered in a Family Health Unit located in the rural area of the municipality of São João do Rio do Peixe, Paraiba. Bardin's content analysis technique was used. RESULTS: From the data obtained in the research, three categories emerged: Women's knowledge about the human papillomavirus (HPV); guidance received from the perspective of women; and forms of prevention used by women against HPV. Understanding about HPV is permeated by the lack of instruction about the virus, although most interviewees recognize condoms as an essential way of prevention, even reporting that they have not received adequate guidance on HPV from the Family Health Unit staff that attends in the community. CONCLUSION: It was identified a deficit in women's knowledge in that rural area about the human papillomavirus, mainly regarding the form of transmission, its causes, and precautions for prevention. It is up to the health team to develop new health education strategies on HPV so that the community has better information related to disease prevention.

DESCRIPTORS: Human papillomavirus. Prevention. Rural population. Women. Nursing. 


\section{Introdução}

O Papilomavírus Humano (HPV) é considerado um vírus, o qual é transmitido principalmente pelo ato sexual, causador de uma infecção sexualmente transmissível (IST), que afeta homens e mulheres, sendo considerado um problema de saúde pública ${ }^{1}$. É um vírus constituído de DNA que tem maior afinidade pelo epitélio cutâneo e mucoso, ocasionando o crescimento anormal de células epiteliais no ser humano e nos animais. Existem cerca de 100 genótipos de HPV, sendo que os tipos 16 e 18 juntos destacam-se por ser considerados de alto risco e causadores de 70\% dos casos de câncer cervical, enquanto o HPV dos tipos 6 e 11 são considerados de baixo risco e podem causar verrugas genitais 2,3 .

No mundo, cerca de 291 milhões de mulheres aprensentam a infecção pelo HPV em alguma fase da vida, equivalendo a um predomínio de $10,4 \%$, sendo que mais de $90 \%$ dessas infecções regridem naturalmente em 6 a 18 meses $^{4}$.

No Brasil, não existem bases estatísticas de prevalência de infecção pelo HPV em indivíduos sexualmente ativos. De acordo com o público examinado e o metódo de diagnóstico utilizado, a incidência nas mulheres varia de 0,7 a $10 \%$. A prevalência varia de $10 \%$ a $50 \%$ entre os 15 e 25 anos, fase de início da prática sexual, sendo esse período o mais propício para a mulher adquirir a infecção, e após os 30 anos, esta prevalência diminui para 3 a $5 \%$ nas mulheres 5 .

Dados afirmam que $50 \%$ a $80 \%$ das infecções ocorrem de dois a três anos após o início da vida sexual, e a população mais acometida corresponde aos adolescentes sexualmete ativos ${ }^{6}$. Já entre as mulheres na faixa etária de 20 a 25 anos, destaca-se como as mais afetadas geralmente aquelas com vida sexual ativa ou com vários parceiros ${ }^{7}$. Apesar de ainda não existir um teste para confirmar a infecção por HPV nos homens, eles são um importante vetor e propagador do vírus, colaborando para o aumento de câncer de colo de útero nas mulheres, expondo-se também à morbidade e à mortalidade por consequência da infecção do HPVํ․
Atualmente, evidenciou-se o aumento da doença infecciosa causada por esse vírus com manifestação assintomática determinada por vários tipos de HPV. A ausência de conhecimento, condições biológicas e conceitos errôneos têm contribuído para a disseminação da IST. As ações de educação em saúde do governo não são voltadas para o HPV, sendo indispensável investigar o nível de informação e práticas sexuais para que as condutas informativas obtenham efeito positivo na vida da comunidade? .

A população tem um entendimento restrito sobre o HPV, devido as informações serem predominantemente vínculadas pela mídia de modo não compreensível para os indivíduos, podendo-se destacar também os serviços de saúde, universidades e profissionais que muitas vezes não orientam adequadamente a população sobre a relevância destes vírus e consequências da infecção. Nesse contexto, a educação em saúde é vista como a construção do conhecimento da enfermagem, do poder público e do cuidado à saúde das pessoas ${ }^{10}$.

Diante da exposição da população ao vírus, a enfermagem pode colaborar na prevenção do HPV e do câncer de colo de útero através de atividades educativas em saúde, informando ao público feminino sobre a importância da realização de exames preventivos regularmente e orientar quanto às outras formas de prevenção do vírus, como a vacinação ${ }^{11}$.

A vacina contra o HPV é um método utilizado para a diminuição dos casos de câncer em mulheres, pois previne lesões cervicais pré-cancerosas do colo do útero, vulva, vagina, câncer do colo do útero e verrugas genitais ocasionadas pelos vírus 6, 11, 16 e 18 12,13.

Nesta perspectiva, sabendo-se que a ausência de educação em saúde sobre a disseminação e prevenção do HPV confere uma temática significativa para a sáude da população em geral, principalmente as mulheres que são mais vulneráveis a adquirir a infecção. Além disso, e tendo em vista que este é um grande fator de risco para o desenvolvimento do câncer de colo de útero, é de extrema importância que mais estudos científicos sobre essa patologia sejam realizados no intuito de despertar no profissional de sáude uma intervenção em educação sexual mais eficaz à população. 
Diante do exposto, este artigo tem como objetivo avaliar o conhecimento das mulheres de uma zona rural sobre o Papilomavírus Humano e formas de prevenção.

\section{Método}

Trata-se de um estudo de natureza descritiva e exploratória, com abordagem qualitativa, realizado numa comunidade da zona rural do município de São João do Rio do Peixe, Paraíba, Brasil. A escolha deste local é justificada através do grande número de mulheres residentes e pela ausência da realização de educação em saúde na comunidade.

A amostra foi composta por 43 mulheres cadastradas na Unidade de Saúde da Família (USF) em questão, delimitadas por forma não probalística e por conveniência. Os critérios de inclusão foram: mulheres acima de 18 anos, estarem presentes no momento da coleta e aceitarem participar da pesquisa.

A coleta de dados occoreu no mês de outubro de 2017 por meio de entrevista semiestruturada. As entrevistas foram realizadas por uma pesquisadora devidamente treinada, a qual foi ao encontro das participantes em suas residências, garantindo assim um ambiente privativo para tal atividade. A duração das entrevistas teve em média 50 minutos, e foi composta de duas partes. A primeira parte foi constituída por coleta de dados de caracterização, como idade, estado civil, escolaridade e atividade sexual. A segunda parte da entrevista foi composta pelas seguintes questões subjetivas que atendem aos objetivos do estudo: A senhora sabe o que é o HPV? Onde obteve informações sobre o HPV?; Quais são os sintomas e o que pode causar?; Como se dá sua transmissão?; Como podemos nos prevenir contra o HPV? ; Qual foi a orientação que a senhora obteve do enfermeiro (a) da USF sobre o HPV?; A senhora sabe que o ministério da saúde oferece a vacina para meninas e meninos contra o HPV?.
Antes de cada entrevista, a pesquisadora forneceu às participantes informações quanto à importância e objetivos da pesquisa. Aquelas que aceitaram participar do estudo assinaram o Termo de Consentimento Livre e Esclarecido (TCLE). As entrevistas foram gravadas com o auxílio de um aparelho mp4 e, posteriormente, transcritas na íntegra para análise da subjetividade.

Os dados foram analisados utilizando a técnica de Bardin ${ }^{14}$, por meio da análise de conteúdo. Para assegurar a privacidade dos participantes, estes foram identificados com letras acompanhadas de números sequenciais correspondentes à ordem que foram realizadas as entrevistas.

Foram obedecidas as orientações inerentes ao protocolo de pesquisa que se encontra na resolução 466/12 do Conselho Nacional de Saúde (CNS), que normatiza as pesquisas envolvendo seres humanos. Este estudo teve a aprovação pelo Comitê de Ética em Pesquisa do Centro Universitário de João Pessoa UNIPÊ com n ${ }^{\circ}$ CAAE 71724017.5.0000.5176.

\section{Resultados}

Participaram do estudo 43 mulheres com idade entre 18 e 49 anos. Destas, 26 tinham de 18 a 35 anos, e 17 entre 36 a 49 anos. Quanto à situação conjugal, 32 eram casadas, 02 divorciadas, 06 solteiras, e 03 encontravam-se em união estável. No que se refere à escolaridade, 02 tinham o ensino fundamental completo, 18 o ensino fundamental incompleto, 11 o ensino médio completo, 08 o ensino médio incompleto e 04 o ensino superior completo ou incompleto. Quanto à atividade sexual, 37 eram ativas e 06 não ativas.

A partir dos dados obtidos na pesquisa, emergiram três categorias: Conhecimento das mulheres sobre o HPV; Orientações recebidas na perspectiva das muIheres; e Formas de prevenção utilizadas pelas muIheres contra o HPV. 


\section{Conhecimento das mulheres sobre o HPV}

Procurou-se investigar o conhecimento que as mulheres têm sobre o HPV, entendendo que o conhecimento é uma ferramenta muito importante para os cuidados com a saúde. Com base nas entrevistas realizadas, identificou-se que a maioria das mulheres tinha um conhecimento insuficiente e/ou fragmentado.

Não é a bactéria ou é o vírus que transmite o HPV? É o que provoca o câncer de colo. (E6)

Acho que é um vírus sexualmente transmissível. (E7)

É um tipo de vírus, são vários tipos de vírus que juntos se denominam papiloma vírus. (E10)

Uma doença que pega no útero quando transa com outra pessoa, crista de galo é?. (E11)

Não sei exatamente a relação, só sei que tem a ver com o surgimento do câncer de colo do útero. (E13)

Já fui informada. É um vírus, não é? Eu já até tive, fiz até tratamento, fiz cauterização de uma feridinha na vagina. (E28)

É vírus que pode virar uma doença grave se não se cuidar. (E37)

Acho que o HPV e o câncer são a mesma coisa, doença sexualmente transmissível. (E40)

Algumas entrevistadas relataram não saber do que se tratava:

Eu tenho um conhecimento e ao mesmo tempo não tenho, porque eu sei que existe esse vírus, mas não sei como ele é causado, não sei o que ele transforma no corpo de uma mulher. (E1)

Sei não. Só sei do câncer do colo do útero porque pesquiso na internet. (E11)

Não sei. O que é HPV? Acho que é uma doença. (E16)

Não sei. (E42)

Não. O HPV não é o câncer do colo do útero não?. (E15)
Outras mulheres possuíam um saber equivocado:

É o vírus da AIDS. (E5)

Acho que são umas verrugas que saem no corpo. (E9)

É o câncer do colo do útero. (E15)

Acho que é a vacina que toma. (E34)

\section{Orientações recebidas na perspectiva das mulheres}

Com relação às orientações recebidas por parte dos profissionais da USF sobre o vírus do HPV, todas as mulheres entrevistadas afirmaram que não receberam nenhum tipo de orientação:

Nenhuma, porque ninguém nunca veio falar sobre isso não. (E11)

Não tive orientação do enfermeiro sobre o HPV. (E12)

No que diz respeito à orientação sobre a importância do exame citológico recebida na consulta de enfermagem, a maior parte das entrevistadas relatou que a única orientação dada pela enfermeira acerca deste exame foi que servia para prevenir doenças. Não ficou perceptível para as entrevistadas uma orientação clara sobre a importância da realização do exame, uma vez que o exame citológico é de fundamental importância na detecção precoce do câncer de colo de útero.

Explica que é para prevenir doenças. (E18)

Eu fiz o exame, mas não me informaram nada não [...] a orientação que diz é que tem que fazer esse exame de ano em ano. (E22)

Eles explicam que tem que estar fazendo todo o ano porque se tiver alguma doença já detecta no exame.

A informação que passaram para mim é que eu tenho que fazer os exames ginecológicos a cada seis meses, pelo menos. (E28)

Não tive informações da USF. (E2)

Quando fosse transar com os parceiros usar camisinha. 
Com relação à obtenção de informações sobre a vacinação contra o Papilomavírus, a maioria das entrevistadas afirmou que foram orientadas pelo agente de saúde. Outras relataram que obtiveram essas informações por meios televisivos, escola ou simplesmente não foram informadas:

Fiquei sabendo pela agente de saúde. (E4)

Pelos comerciais de TV e pelo agente de saúde que passa nas casas avisando das campanhas. (E24)

Eu só escuto falar no HPV quando tem essas campanhas de vacinação. Por meio da televisão, e às vezes algumas campanhas na cidade você acaba vendo cartazes. (E21)

Fui informada não, eu vi passando na TV. (E27)

Sim, fiquei sabendo através da escola. (E9)

Pelos jornais. (E29)

Fui informada porque os meninos daqui foram tomar as vacinas. (E17)

\section{Formas de prevenção utilizadas pelas mulheres contra o HPV}

Nesta categoria buscou-se analisar as formas de prevenção conhecidas e utilizadas pelas mulheres contra o HPV. No que se refere ao conhecimento sobre as formas de prevenção do HPV, foi identificado que grande parte destas mulheres reconhece o preservativo como uma importante maneira de prevenção, embora um número considerável não soubesse dizer como se prevenir. Poucas mulheres referiram outros meios de prevenção:

Não sei. (E17)

Usando o preservativo. (E7)

Usar camisinha, tomar vacina. (E2)

Fazendo os exames de rotina ginecológicos, usando também o preservativo é uma maneira de evitar. (E28)

Só a vacinação que divulgaram. (E10)

Nas relações sexuais usando a camisinha, não ter tantos parceiros que não usa camisinha e ter cuidado com manipulação de sangue contaminado quem trabalha na área da saúde. (E5)

\section{Discussão}

A maior parte das mulheres submetidas à pesquisa sobre o conhecimento do HPV demostrou que não tem instrução sobre o vírus, e que muitas delas, por não saberem o que é o vírus, acabam confundindo com outras doenças. Tal resultado coincide com o que foi obtido em outro estudo 1 , o qual evidenciou que a maior parte dos jovens já ouviu falar do HPV devido à relevância das IST, porém estes têm mais conhecimentos sobre o vírus da Imunodeficiência humana (HIV) do que sobre o HPV.

Embora esse vírus não seja precisamente tão divulgado e o conhecimento sobre ele se torne insatisfatório e, muitas vezes, até inexistente ${ }^{1}$, sabe-se que atualmente em $100 \%$ das ocorrências de câncer do colo do útero são encontrados o HPV e a maior parte destas pessoas apresenta a infecção transitória e sem sintomas aparentes, dificultando a detecção entre o período de um a dois anos, favorecendo assim a progressão de lesões pré-cancerosas e neoplásicas ${ }^{15}$. Neste contexto, destaca-se que a ausência de informações apropriadas sobre o HPV pode levar a uma falha que prejudica tanto as pessoas afetadas pelo vírus, como também as que participam do seu cenário social.

A compreensão sobre o vírus é uma das principais ferramentas relacionadas à sua prevenção ${ }^{16}$. Nesse sentido, o nível educativo agregado a outras características sociodemográficas precisa ser levado em consideração durante as orientações prestadas às mulheres sobre o vírus, com vista às realizações de estratégias individuais que possibilitem a assimilação das orientações recebidas.

No que se refere ao conhecimento sobre as formas de prevenção do HPV, foi identificado que grande parte destas mulheres reconhece o preservativo como uma importante maneira de prevenção, embora um número considerável não soubesse dizer como se prevenir, bem como poucas mulheres referiram outros meios de prevenção. Este resultado coincide com o encontrado em um estudo semelhante realizado em Recife, mostrando com os depoimentos que os participantes reconhecem o uso do preservativo e a vacinação como estratégias de prevenção eficazes ${ }^{17}$.

O HPV é evidenciado como um relevante problema de saúde pública diante da letalidade e da alteração do estado de saúde associado às lesões, aliado ao evento do câncer cervical, e por meio de outras patologias ${ }^{18}$. 
A prevenção deste vírus é uma importante estratégia para a diminuição da infecção, sendo essencial a implantação de projetos para esclarecimento sobre os danos associados à infecção, a compreensão das pessoas sobre a importância da prevenção por intermédio da vacinação, recomendações adequadas sobre práticas sexuais e exames de detecção ${ }^{11}$.

No presente estudo, todas as mulheres relataram não terem recebido nenhum tipo de orientação sobre o HPV pelos profissionais de saúde da USF, evidenciando falhas na educação em saúde. A falta de informação acerca do vírus corrobora com um estudo realizado numa escola pública de ensino fundamental e médio no município de Niterói- RJ, mostrando que a ausência do conhecimento a respeito do HPV e do preventivo deve-se a deficiência de conhecimento dos profissionais de saúde e dos serviços sobre o tema ${ }^{19}$. O enfermeiro como um dos membros de uma equipe multidisciplinar na Estratégia Saúde da Família está diante de atividades educativas do SUS e, em conjunto com a comunidade escolar, podem-se elaborar ideias diferenciadas, de acordo com as que foram descritas pelos entrevistados. Ambos têm a competência para instruir corretamente sobre hábitos sexuais entre jovens e na busca ativa precoce de casos suspeitos do vírus ${ }^{20}$.

A educação em saúde é um meio científico utilizado pelos profissionais que favorece o entendimento do processo saúde-doença para a adesão de novas práticas e comportamentos de saúde. O método de instrução é um elemento relevante da ocupação profissional da enfermagem, tendo o propósito de motivar transformações de hábitos e adequações às novas situações de vida da sociedade ${ }^{21}$. Neste contexto, é necessário que os profissionais de saúde sejam capacitados e atualizados periodicamente para que possam fornecer orientações preventivas adequadas à população sobre o HPV.

Considerando que um número reduzido das participantes revelou ter uma concepção clara de que o exame citológico é um exame preventivo do câncer de colo do útero, evidencia insuficiência da orientação sobre a finalidade e a importância da realização deste exame. Um estudo semelhante realizado com dez mulheres numa USF do município de Montes Claros - MG constatou que as orientações durante as consultas de enfermagem não foram direcionadas à prevenção do câncer do colo do útero, mas voltadas apenas para a coleta do exame citopatológico ${ }^{22}$.

O enfermeiro atuante na estratégia de sáude da família tem competência para ajudar a prevenir o câncer cervical motivando a realização do exame preventivo através de coleta de material do exame citológico, na qual é responsável por identificar as mulheres em situações de risco ${ }^{23}$. A consulta de enfermagem é designada como uma atribuição do enfermeiro que serve para identificar as adversidades do processo saúdedoença, efetuando e analisando os cuidados que colaborem para a promoção, proteção, recuperação e reabilitação da saúde ${ }^{24}$. Neste contexto, observa-se que o enfermeiro deve escutar e instruir a mulher antes da coleta do exame, de maneira a acalmá-la. É importante que o profissional seja capacitado para saber lidar com as barreiras culturais impostas, de maneira que possa explicar a importância do exame, esclarecendo a relação do HPV com o câncer do colo do útero.

Os agentes comunitários de saúde tiveram destaque nos discursos das informantes deste estudo, visto que eles também fazem parte da equipe de saúde responsável pela divulgação da vacinação contra o HPV dentro da faixa etária designada pelo Ministério da Saúde. Em relação a termos de conhecimentos das mulheres sobre a vacinação, e comparando-se com um estudo realizado em Campinas-SP, a vacinação era conhecida por menos de $9 \%$ dos entrevistados no ano de 20119, enquanto que em outro estudo, desenvolvido no ano de $2014,75,8 \%$ das mulheres conheciam a vacina contra o HPV25. É importante ressaltar que a vacina não substitui a realização do exame de Papanicolau, porém corresponde a um instrumento de prevenção primária quando induz a regressão de lesões precursoras e remissão do câncer de colo de útero. A realização do exame preventivo é de grande importância, visto que as vacinas não dão proteção para todos os tipos de vírus do HPV, sendo este exame responsável pela diminuição dos casos de câncer de colo do útero em países desenvolvidos 3 .

Portanto, é de extrema importância que todos os profissionais de saúde disseminem informações sobre a vacinação, ressaltando que a imunização é crucial na prevenção do HPV, mas que apenas esta não é capaz de fornecer uma proteção total, sendo importante associar a prevenção com o uso do preservativo nas relações sexuais e a realização periódica do exame citológico. 


\section{Conclusão}

Este estudo possibilitou identificar o déficit de conhecimento das mulheres da referida zona rural sobre o papilomavírus humano, principalmente no que tange a forma como é transmitido, suas causas e os cuidados que devem ser tomados quanto à prevenção. A ausência de conhecimento sobre a finalidade e importância do exame citológico na prevenção e detecção do vírus do HPV prejudica as práticas de autocuidado e prevenção do câncer do colo do útero também foi relatado entre as participantes.

Tendo em vista o déficit de informações sobre o HPV nesta comunidade, cabe à equipe de saúde, especialmente ao enfermeiro, desenvolver novas estratégias de educação em saúde para a população, informando adequadamente sobre o HPV e seus agravos, ressaltando as formas de prevenção disponíveis, tanto contra esta quanto a outras doenças sexualmente transmissíveis e também evidenciando a importância do exame preventivo.

\section{Contribuições das autoras}

Abreu LS participou do desenvolvimento da pesquisa, concepção, delineamento, coleta de dados, interpretação dos resultados, e redação do artigo científico. Andrade TSO participou da coleta de dados e redação do artigo científico. Nunes ZM participou da redação do artigo científico. Rufino NS, coorientadora e Martins KP orientadora e supervisora da pesquisa, participaram da concepção, delineamento, interpretação dos resultados e redação.

\section{Conflitos de interesses}

Nenhum conflito financeiro, legal ou político envolvendo terceiros (governo, empresas e fundações privadas, etc.) foi declarado para nenhum aspecto do trabalho submetido (incluindo, mas não se limitando a subvenções e financiamentos, participação em conselho consultivo, desenho de estudo, preparação de manuscrito, análise estatística, etc.).

\section{Referências}

1. Sepúlveda-Carrillo GJ, Goldenberg P. Conhecimentos e práticas de jovens sobre a infecção pelo papilomavírus humano: uma questão re-atualizada. Rev Colomb Obstet Ginecol. 2014;65(2):152-61. https://doi.org/10.18597/rcog.63
2. Rama CH, Villa LL, Pagliusi S, Andreoli MA, Costa MC, Aoki AL, et al. Awareness and knowledge of HPV, cervical cancer, and vaccines in young women after first delivery in São Paulo, Brazil a cross-sectional study. BMC Womens Health. 2010;10:35. https:// doi.org/10.1186/1472-6874-10-35

3. Libera LSD, Alves GNS, Souza HG, Carvalho MAS. Avaliação da infecção pelo Papiloma Vírus Humano (HPV) em exames citopatológicos. Revista Brasileira de Análises Clínicas. 2016;48(2):138-43. Disponível em: http://www.rbac.org.br/artigos/ avaliacao-da-infeccao-pelo-papiloma-virus-humano-hpv-emexames-citopatologicos-48-n2/

4. Ministério da Saúde (Brasil). Instituto Nacional do Câncer. Estimativa 2016: incidência de câncer no Brasil [Internet]. Rio de Janeiro (RJ): INCA; 2015. Disponível em: https:// santacasadermatoazulay.com.br/wp-content/uploads/2017/06/ estimativa-2016-v11.pdf

5. Hpv info Brasil. Hpv livro: 1. Epidemiologia do hpv [Internet]. [citado em 2017 mar 15]. Disponível em: http://hpvinfo.com.br/ hpv-livro-1-epidemiologia-do-hpv

6. Roteli-Martins CM, Longatto AF, Hammes LS, Derchain SFM, Naud P, Matos JC. Associação entre idade ao início da atividade sexual e subsequente infecção por papilomavírus humano: resultados de um programa de rastreamento brasileiro. Rev Bras Ginecol Obstet. 2007; 29(11):580-7. https://doi.org/10.1590/50100$\underline{72032007001100006}$

7. Luz NNN, Lustosa ÍR, Machado KC, Pacheco ACL, Marques MMM, Peron AP, et al. Acadêmicos, a percepção sobre o papilomavírus humano e sua relação com o câncer cervical. Semina Ciências Biológicas e da Saúde. 2014;35(2):91-102. http:// dx.doi.org/10.5433/1679-0367.2014v35n2p91

8. Santos IM, Maioral MF, Haas P. Infecção por HPV em homens: Importância na transmissão, tratamento e prevenção do vírus. Estud Biol. [Internet]. 2011;32/33(76-81):111-8. https:// periodicos.pucpr.br/index.php/estudosdebiologia/article/ download/22877/21980

9. Osis MJD, Duarte GA, Sousa MH. Conhecimento e atitude de usuários do SUS sobre o HPV e as vacinas disponíveis no Brasil. Rev Saúde Pública. 2014;48(1):123-33. http://dx.doi.org/10.1590/ S0034-8910.2014048005026

10. Silva LAA, Schmidt SMS, Signor E, Noal HC, Gomes IEM. Avaliação da educação permanente no processo de trabalho em saúde. Trab Educ e Saúde. 2016;14(3):765-81. http://dx.doi. org/10.1590/1981-7746-sol00015

11. Souza SV, Ponte KMA, Júnior DGA. Prevenção do HPV nas mulheres: estratégia adotada por enfermeiros na atenção primária à saúde. Sanare. [Internet]. 2015;14(01):46-51. https:// sanare.emnuvens.com.br/sanare/article/view/607/324 
12. Calumby RJN, Silva RA, Suárez JAG, Lôbo TDLGF, Vieira DS, Silva KWL et al. Papiloma Vírus Humano (HPV) e neoplasia cervical: importância da vacinação. Brazilian Journal of Health Review. 2020; 3(2):1610-1628. https://doi.org/10.34119/bjhrv3n2-023

13. Thomas TL. Cancer Prevention: HPV Vaccination. Semin Oncol Nurs. 2016;32(3):273-80. https://dx.doi.org/10.1016\%2Fj. soncn.2016.05.007

14. Bardin L. Análise de conteúdo. $6^{a}$ ed. São Paulo (SP): Almedina; 2011.

15. Andrade MS, Almeida MMG, Araújo TM, Santos KOB. Fatores associados a não adesão ao Papanicolau entre mulheres atendidas pela Estratégia Saúde da Família em Feira de Santana, Bahia, 2010. Epidemiol Serv. Saúde. [Internet] 2014;23(1):11120. https://www.scielo.br/pdf/ress/v23n1/2237-9622ress-23-01-00111.pdf

16. Oliveira AL, Ferreira JB, Miranda VC, Morais KCS. Papilomavírus humano: conhecimento feminino sobre a prevenção. Rev Pesqui Fisioter. 2017;7(2):179-87. http://dx.doi.org/10.17267/22382704rpf.v7i2.1301

17. Silva PMC, Silva IMB, Interaminense INCS, Linhares FMP, Serrano SQ, Pontes CM. Conhecimento e atitudes sobre o Papilomavírus humano e a vacinação. Esc Anna Nery. 2018;22(2):1-7. http://dx.doi.org/10.1590/2177-9465ean-2017-0390

18. Soares MAD. O conhecimento dos acadêmicos de Enfermagem acerca das medidas preventivas do HPV junto à população feminina [monografia]. Niterói: Universidade Federal Fluminense; 2015. https://app.uff.br/ riuff/bitstream/1/973/1/0\%20conhecimento $\% 20$ dos $\% 20$ acad\%C3\%AAmicos\%20de $\% 20$ enfermagem $\% 20$ acerca $\% 20$ das $\% 20$ medid.pdf

19. Barbeiro FMS, Cortez EA, Oliveira PAMC, Silva ALO. Conhecimentos e práticas das mulheres acerca do exame papanicolau e prevenção do câncer cérvico-uterino. Rev de Pesq cuidado é fundamental online [Internet]. 2009;1(2):41422. Disponível em: http://www.seer.unirio.br/index.php/ cuidadofundamental/article/view/419
20. Souza G, Alves PS. Estratégias educativas para prevenção e redução da morbimortalidade do câncer do colo uterino. Saúde e Pesquisa [Internet]. 2015;8(2):317-26. Disponível em: https:// periodicos.unicesumar.edu.br/index.php/saudpesq/article/ view/4258

21. Souza AF, Costa LHR. Conhecimento de mulheres sobre HPV e câncer do colo do útero após consulta de enfermagem. Rev Bras Cancerol. 2015;61(4):343-50. https://doi.org/10.32635/2176-9745. $\underline{\text { RBC.2015v61n } 4.220}$

22. Nogueira KRC, Moraes MM. Prevenção do câncer cervical: o conhecimento das usuárias em uma equipe de saúde da família. Rev Enferm UFPE online [Internet]. 2017;11(5):1892-901. Disponível em: https://pesquisa.bvsalud.org/enfermeria/resource/ pt/biblio-1032130

23. Pereira RTA, Ferreira V. A consulta de enfermagem na estratégia saúde da família. Rev Uniara. 2014;17(1):99-111. https://doi.org/10.25061/2527-2675/ReBraM/2014.v17i1.10

24. Freitas RA, Cangussú LV, Gradella DBT. Conhecimento de mulheres usuárias do SUS sobre o HPV na região norte do Espírito Santo. Enciclopédia Biosfera. 2015;11(22):3239-48. http://dx. doi. org/10.18677/Enciclopedia Biosfera 2015040

25. Jalani FFM, Rani MDM, Isahak I, Aris MSM, Roslan N. Knowledge, Attitude and Practice of Human Papillomavirus (HPV) Vaccination among Secondary School Students in Rural Areas of Negeri Sembilan, Malaysia. Int J Collab Res Intern Med Public Health [Internet]. 2016;8(6):420-34. Disponível em: https://internalmedicine.imedpub.com/knowledge-attitudeand-practice-of-human-papillomavirus-hpv-vaccinationamong-secondaryschool-students-in-rural-areas-of-negeri-se. php?aid=9905 